\section{An Interactive Data System for Physicists}

\section{F. J. Smith, Belfast}

Too much of the time of the average physicist is spent carrying out routine operations, such as extracting data from references and changing units. In the process, he often makes minor simple arithmetic errors which cost him much wasted effort later. Yet these activities are well within the capabilities of a small or moderate computer system at a low cost, if the software was available. If a fraction of the effort put into developing on-line airline reservation systems was put into the development of online data retrieval and data manipulation systems for scientists, it is possible that such systems would be more cost effective than the airline systems because of the time saved by the scientist and because of his extra productivity. Unfortunately, the time of a physicist is not valued as it should be, as it does not appear as a direct cost in any accounts and because his work is longer term than that of most others; so, not enough thought and effort (and money) is given to increasing his productivity. Our work in Belfast is aimed at studying one aspect of this problem : seeing how on-line data systems can be of help in the future.

To investigate the problem we have selected one area of Atomic and Molecular Physics - a study of the interaction energies between two atoms. We have searched through all of the literature since 1965 until the present and stored on a disc file all of the useful information on interatomic potentials we have found. This has involved extracting from the literature and reading in detail some 1400 papers to date (February, 1973) and recording in our file the following :

1 Paper title and reference

2 Diatomic system and state name

3 Type of potential

4 Method of calculation

5 Parameters, formula or values which actually define the potential

6 Units

7 Error and source of error

8 Range

9 Any other relevant information.

\section{Retrieval and Manipulation}

The operation of the pilot system requires little or no knowledge on the part of the user. He communicates by on a teleprinter linked to the computer over the telephone network. The system in its present form allows for the retrieval of any of the stored potentials. As well as the range and accuracy of the data reported, the units (stored as atomic units) can be changed to suit the needs of the user. In addition he can have an off-line graph of the potential, should he require it.

However, a scientist is not just interested in the potential itself, but rather he usually wants it to help him calculate parameters defining one of the physical or chemical properties of matter. Hence, we are presently including with the system programs to help him make such calculations. Examples already in existence include those for calculating deflection angles, phase shifts and, shortly, also the transport properties of gases.

\section{The Curve-fitting Problem}

To enable calculations to be made with a particular potential, an automatic procedure is required to construct, from the various approximations of the potential which have been stored, an estimated potential which is representative of the most reliable results available. Our present aim is to set up a system whereby this curve can be obtained from the available data describing the interatomic potential. The trouble is that the raw data for the potentials, as it appears in the literature, take a number of forms such as algebraic formulae, for example, $V(r)=a e^{-b r}$ where $a$ and $b$ are constants, as curves on published graphs or as tables of values. There are many ways of determining the potentials both by experimental results and also by means of theoretical calculations, with the result that the information available on a particular potential may vary enormously in accuracy and range of validity. Hence, first and foremost, a good method of evaluating the data is mandatory we hope to involve the assistance of consultants to overcome this side of the problem. answering multiple-choice questions

\section{The "Potential" User}

We hope to encourage the future use of our system by allowing users not only to avail themselves of a fixed number of operations that we have incorporated but also to extract and, if necessary, to manipulate the data by storing it in their own personal files, and to apply their own suite of programs, for whatever purpose.

\section{Example}

In conclusion, may I suggest a conversation between a physicist of the future (1980?) with his terminal beside his desk.

Physicist presses the log-in button on his terminal

$\begin{array}{ll}\text { Local computer: } & \text { Ready } \\ \text { Physicist: } & \text { Information } \\ \text { Local computer: } & \text { Subject? } \\ \text { Physicist: } & \text { Atomic and } \\ & \text { Molecular Physics }\end{array}$

Local computer : You will be connected to the data bank in Belfast

Belfast: $\quad$ Are you familiar with the Belfast data system on Atomic and Molecular Physics?

Physicist: Yes

Belfast : References or Data?

Physicist: Data

Belfast : Subject?

Physicist: Interatomic Potential

Belfast :

Physicist :

Belfast :

Physicist :

Belfast :

Atoms or ions?

$\mathrm{H} \quad \mathrm{H}$

State?

Physicist :

Belfast :

Physicist :

Belfast :

Physicist :

Belfast :

Triplet sigma $U$

All data or best data available?

Best

Units?

eV, a.u.

Range?

?

State range of internuclear distance for which your interatomic potential is required in your units. Type two numbers separated by a comma giving the lower and upper limits required.

\section{Range}

Belfast: Form ?

Physicist : Graph

\section{Belfast: By post?}

Physicist: Yes

Belfast: $\quad$ Please type the name and address to which graph will be posted to-morrow. etc. 